\title{
Dermatopathy in juvenile Angus cattle due to vitamin A deficiency
}

Journal of Veterinary Diagnostic Investigation 24(4) 763-766

(C) 2012 The Author(s)

Reprints and permission: sagepub.com/journalsPermissions.nav DOI: $10.1177 / 1040638712445767$

http://jvdi.sagepub.com

\section{Thomas J. Baldwin, ${ }^{1}$ Kerry A. Rood, E. Jane Kelly, Jeffery O. Hall}

\begin{abstract}
In juvenile cattle, vitamin A deficiency is reported most commonly as a neurological condition; only rarely are there dermatologic manifestations. In the current study, alopecia, severe epidermal and follicular orthokeratosis, and acanthosis due to hypovitaminosis A are reported in 2 of 32 Angus calves, with a third animal suspected. Affected animals responded to vitamin A supplementation, and no additional calves displayed signs. Vitamin A acts on skin by regulating DNA transcription in keratinocytes, reducing the number of tonofilaments and desmosomes, both involved in cell-to-cell adhesion. Hence, adequate levels of dietary vitamin A are necessary for normal keratinocyte turnover, and deficiencies result in retention of keratinized cells (orthokeratosis). The present report reminds diagnosticians to consider vitamin A deficiency in cases of orthokeratotic dermatopathy in cattle.
\end{abstract}

Key words: Angus cattle; dermatopathy; hypovitaminosis A; orthokeratosis; vitamin A deficiency.

Vitamin A deficiency in juvenile cattle is associated commonly with neurologic signs attributed to defective remodeling of membranous bone, which leads to compression of optic and other nerves and increases in cerebrospinal fluid pressure. ${ }^{5,15,20}$ Night and complete blindness, ataxia, and convulsions are reported clinical signs. ${ }^{1,22}$ Dermatologic manifestations of vitamin A deficiency are described in cattle, pigs, cats, and $\operatorname{dogs}^{14}$ but are rarely reported in the veterinary medical literature. In cattle, cutaneous lesions consist of a severe scaling dermatitis. ${ }^{14}$ In addition, squamous metaplasia of the mucus glands of the esophagus and other secretory epithelia, plus hyperkeratosis of the esophageal lining, is reported. $^{2,22}$ In cattle, squamous metaplasia of the parotid salivary gland interlobular ducts is considered pathognomonic for vitamin A deficiency. ${ }^{15,22}$

Cattle, as well as other animals and human beings, are incapable of de novo synthesis of vitamin A, which consequently must be obtained from dietary sources. ${ }^{13}$ In cattle, vitamin $A$ is obtained primarily from $\beta$-carotene found in green forages. ${ }^{21}$ Once within enterocytes, most $\beta$-carotene is cleaved at the $15,15^{\prime}$ central double bond to form 2 molecules of retinal, the aldehyde form of vitamin A, which is subsequently reduced to the alcohol form, retinol. ${ }^{4}$ Additionally, some $\beta$-carotene is incorporated with dietary lipids into nascent chylomicrons. ${ }^{4}$ From enterocytes, vitamin $\mathrm{A}$ is transported via the vasculature primarily to, and then stored within, the liver, where it is available for use during times of dietary insufficiency.

In beef cattle, vitamin A deficiency occurs when animals are pastured during periods of drought or fed unsupplemented (vitamin A) rations of grain or hay. ${ }^{21,22}$ Hepatic stores are typically adequate to carry animals through short periods (months) of dietary insufficiency, but longer periods of deprivation result in clinical signs. ${ }^{21}$ Because calves are metabolically more active than adult animals and have reduced stores of vitamin A, they are more susceptible to deprivation. ${ }^{5}$ Thus, signs of deficiency have been reported in calves, whereas older herd mates remain unaffected. ${ }^{15}$ In feedlot cattle, clinical signs occur most often in younger animals fed a diet deficient in vitamin A for 6 to 12 months. ${ }^{21}$ Steers are affected more often than heifers, which may be due to provision of vitamin A by the corpus luteum. ${ }^{20}$

In October 2009, two 8-month-old, female Angus calves, each weighing approximately $170 \mathrm{~kg}$, with a history of progressive dermatitis and deteriorating overall health were referred for veterinary examination. Duration of illness was estimated between 5 and 6 months. A third calf was reported as being affected similarly but had been culled from the herd previously. The 3 calves (out of 32 born earlier that year) were part of a small cow-calf operation. Affected calves were female. Two bulls were used to service the herd's 40 cows, but whether or not the same bull sired the affected animals is unknown.

The herd was kept on 750 acres of western Juniper forest contiguous with 250 acres of grass pasture. Drought conditions were prevalent the preceding spring and summer, resulting in reduced quantity and quality of available forage. Supplemental alfalfa hay was provided to the herd during the previous late fall and winter months but not during spring

From the Utah Veterinary Diagnostic Laboratory, Logan, UT (Baldwin, Kelly, Hall), and the Department of Animal, Dairy and Veterinary Sciences, Utah State University, Logan, UT (Baldwin, Rood, Kelly, Hall).

${ }^{1}$ Corresponding Author: Thomas J. Baldwin, Utah Veterinary Diagnostic Laboratory, 950 East 1400 North, Logan, UT 84341. tom.baldwin@usu.edu 


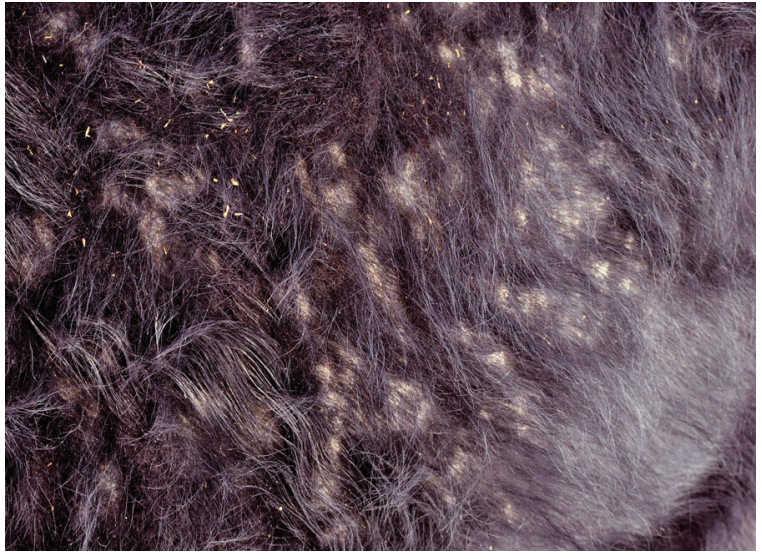

Figure 1. Skin over right lateral abdominal wall with multifocal to coalescing areas of thickening, scaling, and alopecia.

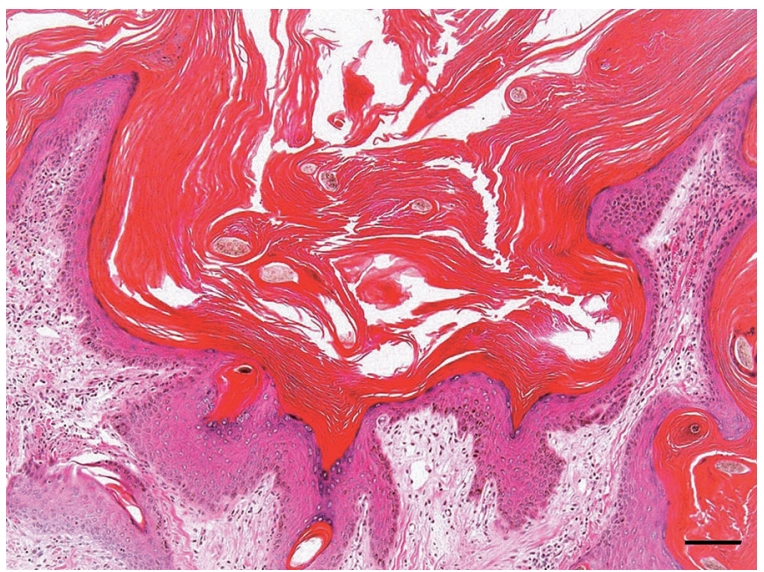

Figure 2. Photomicrograph of skin from a clinically affected Angus calf. Severe orthokeratosis thickens the stratum corneum. Hematoxylin and eosin stain. Bar $=100 \mu \mathrm{m}$.

and summer. A commercially available protein and mineral supplement lacking vitamin A was available free choice.

One of the submitted calves was necropsied immediately following humane euthanasia, whereas a skin sample was obtained surgically from the other and examined histopathologically. The calf from which the skin biopsy was taken was returned to the herd. In the necropsied animal, the skin of the head, neck, tail base and proximal tail, and dorsal and dorsolateral body had multifocal to coalescing areas of severe thickening, scaling, and alopecia (Fig. 1). Histopathologically, affected skin had diffuse, severe orthokeratosis (Fig. 2) with occasional globular aggregates of inspissated serum and degenerate neutrophils entrapped between keratin deposits. Dense orthokeratin filled the ostia of multiple telogen follicles. The underlying stratum spinosum was mildly hyperplastic. Hair follicles were in all stages and normal numbers. Edema fluid and small numbers of scattered to perivascular mast cells, neutrophils, lymphocytes,

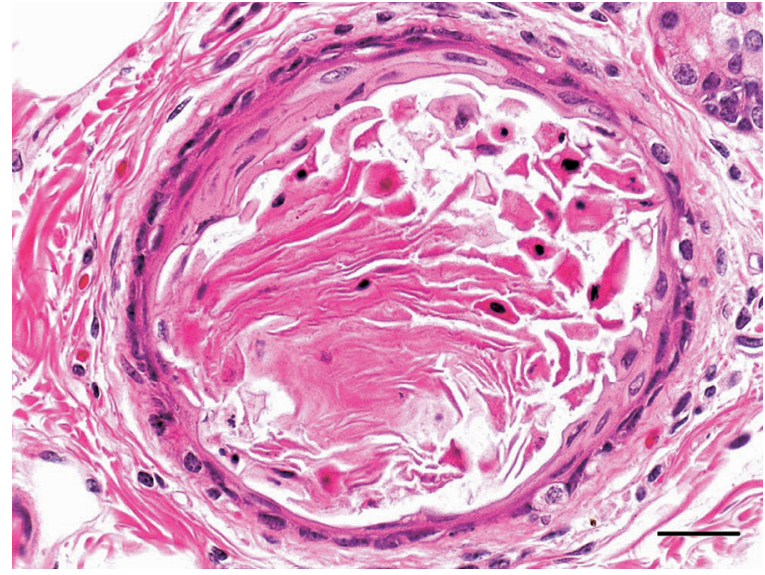

Figure 3. Squamous metaplasia and desquamation of keratin in a parotid salivary gland intralobar duct. Hematoxylin and eosin stain. Bar $=25 \mu \mathrm{m}$.

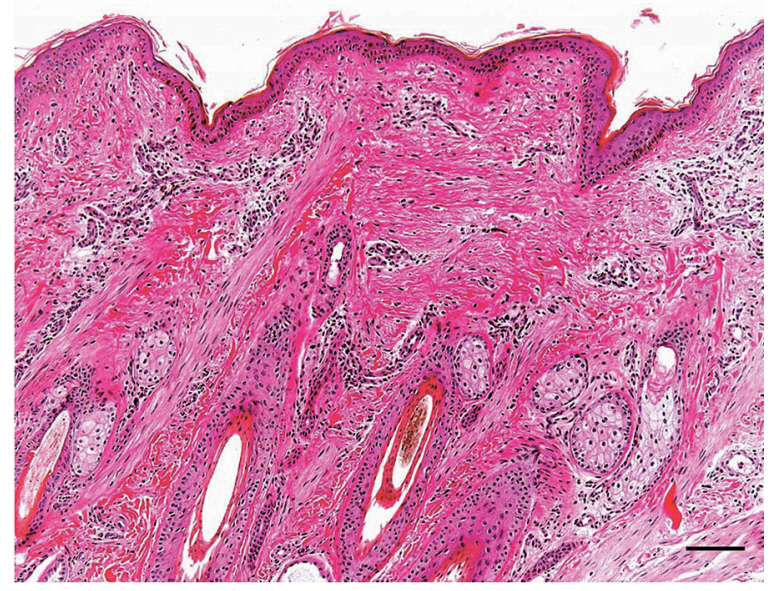

Figure 4. Photomicrograph of skin taken from the same animal and general area as skin sampled and shown in the photomicrograph provided as Figure 2 but obtained 5 months after initiation of vitamin A supplementation. The orthokeratosis has resolved. Hematoxylin and eosin stain. Bar $=100 \mu \mathrm{m}$.

plasma cells, and eosinophils infiltrated the superficial dermis (mild coexisting hypersensitivity response). In addition, in excretory ducts of the parotid salivary gland, stratified squamous epithelium replaced the normal pseudostratified epithelium (squamous metaplasia), and keratinized exfoliated epithelial cells partially filled duct lumens (Fig. 3). No intestinal, hepatic, ocular, or musculoskeletal pathology was noted, either grossly or by histopathologic examination. In the biopsied skin specimen from the second calf, changes were similar to those in the skin of the necropsied animal.

Hyperkeratosis secondary to either trauma or the observed dermatitis was considered improbable based on lesion distribution and severity. Trauma presents typically as solitary hyperkeratotic foci at pressure points, whereas 
hypersensitivity dermatitis results in hyperkeratosis at sites of repeated self-trauma; both induce notably less severe lesions than seen in calves in the current study.

Exposure to chlorinated naphthalene, either by ingestion or cutaneous absorption, results in a sharp decline of vitamin A in the blood. Over several weeks, this manifests clinically as a condition known as bovine hyperkeratosis. ${ }^{19}$ As this toxicity is hypovitaminosis A related, skin lesions are similar to those reported herein but often more profound. ${ }^{17}$ Chlorinated naphthalene toxicity is rare, as responsible compounds have not been used in farm or food processing equipment since the early $1950 \mathrm{~s} .{ }^{19}$ In addition to reported skin lesions, squamous plaques develop in the oral cavity and esophagus; intestinal and biliary epithelial hyperplasia are present with cyst formation, renal tubular hyperplasia, and dilation; and foci of uterine mucosal squamous metaplasia are reported. ${ }^{17}$ None of these non-skin-related lesions were identified in the calf submitted for postmortem examination or by clinical examination of herd mates, arguing against chlorinated naphthalene toxicity as the cause of hyperkeratosis in these cattle.

Secondary hypovitaminosis A, due to chronic diseases of the liver or intestines, can result in absorption or storagerelated deficiencies in vitamin A even when dietary levels of $\beta$-carotene are adequate because most of the conversion of $\beta$-carotene to vitamin A occurs in the intestinal epithelium, and the liver is the principle storage site of this vitamin. ${ }^{4}$ No animals in the affected cow-calf operation displayed signs of enteritis or hepatitis, and the intestine and liver were normal in the animal examined postmortem. Consequently, secondary vitamin A deficiency was considered an unlikely cause of observed hyperkeratosis.

Given the clinical, gross, and histopathologic findings, vitamin A analysis using high-performance liquid chromatography was conducted on the liver from the necropsied calf. Vitamin A was identified at 1.35 parts per million (ppm; normal range for calf liver $=75-150 \mathrm{ppm}$ ). A diagnosis of dermatopathy due to vitamin A deficiency was made and provided to the owner.

Vitamin A was given to the affected and other calves using a commercially available injectable product containing $500,000 \mathrm{IU}$ of vitamin A and 75,000 IU of vitamin D per ml. Injections were given 3 times following the timing and dosage schedule provided on the product label. In addition, the free choice protein/mineral supplement was changed to a supplement containing 36,400 IU vitamin A/kg. Over the next several months, the surviving calf's clinical appearance improved steadily, and by late March 2010 (5 months following treatment), a follow-up skin biopsy obtained from the same general location as the earlier sample showed resolution of the hyperkeratosis (Fig. 4). Moreover, over this same time period, no additional calves displayed clinical signs, supporting the original diagnosis of hypovitaminosis A-induced dermatopathy.
Vitamin A acts on skin by affecting transcription of DNA in keratinocytes and consequently protein synthesis. ${ }^{12}$ Retinoic acid, derived by oxidation of retinol, is transported to the nucleus bound to cytosolic retinoid-binding proteins. ${ }^{9}$ Once within the nucleus, retinoic acid binds to receptor proteins that are members of a large superfamily of ligandactivated transcription factors. ${ }^{9}$ Retinoic acid-receptor protein dimers bind to regulatory areas on chromosomes and induce or suppress gene transcription. ${ }^{11}$

In skin, vitamin A-induced regulation of gene transcription results in altered keratin synthesis, decreases in numbers of tonofilaments and desmosomes, heightened disorganization of the stratum corneum, and deposition of amorphous material both within and between keratinocytes. ${ }^{8-10}$ Tonofilaments form part of the keratinocyte cytoskeleton and converge at desmosomes and hemidesmosomes, contributing to intercellular adhesion. ${ }^{6}$ Hence, loss of tonofilaments and desmosomes results in diminished cell-to-cell adhesion. ${ }^{16}$ Disorganization of the stratum corneum contributes further to the loosening of cornified keratinocytes, whereas accumulations of amorphous material have been shown to increase skin fragility, as fiction-induced blisters occur predominately through these deposits. ${ }^{10}$

In addition, vitamin A results in keratinocyte proliferation, leading to mild acanthosis. Specifically, vitamin A stimulates division of basal keratinocytes, both in epidermis and hair follicles. ${ }^{12}$ This produces increased numbers of suprabasilar keratinocyte layers (acanthosis). In addition, vitamin A elevates glycogen content and numbers of polysomes in keratinocytes, reflecting increased metabolic activity. ${ }^{9}$ Given the above, the overall effect of gene regulation by vitamin $\mathrm{A}$ is increased keratinocyte turnover. ${ }^{16}$

In the calves in the current study, vitamin A supplementation led to normal exfoliation of orthokeratotic layers and replacement of overly adherent surface keratinocytes. Over a period of several months, the dermatopathy resolved completely, and no other clinical signs associated with hypovitaminosis A, including neurologic signs or night blindness, were noted.

The present case study serves as a reminder of the many physiologic functions of vitamin A. Retinal is a component of rhodopsin, the light-detecting molecule within rod and cone cells of the retina. ${ }^{18}$ Moreover, through its modulation of DNA transcription, vitamin A enhances immunity, ${ }^{7}$ contributes toward fetal development, ${ }^{3}$ and aids in bone remodeling. ${ }^{15}$ Dermatologic manifestations of hypovitaminosis A are reported uncommonly in the veterinary medical literature, and the current report reminds diagnosticians to consider vitamin A deficiency in cases of orthokeratotic dermatopathy in cattle.

\section{Declaration of conflicting interests}

The author(s) declared no potential conflicts of interest with respect to the research, authorship, and/or publication of this article. 


\section{Funding}

The author(s) received no financial support for the research, authorship, and/or publication of this article.

\section{References}

1. Booth A, Reid M, Clark T: 1987, Hypovitaminosis A in feedlot cattle. J Am Vet Med Assoc 190:1305-1308.

2. Brown CC, Baker DC, Barker IK: 2007, Alimentary system. In: Jubb, Kennedy, and Palmer's pathology of domestic animals, vol. 2, ed. Maxie MG, 5th ed., pp. 35-36. Elsevier, Philadelphia, PA.

3. Cvekl A, Wang WL: 2009, Retinoic acid signaling in mammalian eye development. Exp Eye Res 89:280-291.

4. D'Ambrosio DN, Clugston RD, Blaner WS: 2011, Vitamin A metabolism: an update. Nutrients 3:63-103.

5. Donkersgoed JV, Clark EG: 1988, Blindness caused by hypovitaminosis A in feedlot cattle. Can Vet J 29:925-927.

6. Drochmans P, Freudenstein C, Wanson JC, et al.: 1978, Structure and biochemical composition of desmosomes and tonofilaments isolated from calf muzzle epidermis. J Cell Biol 79:427-443.

7. Duriancik DM, Lackey DE, Hoag KA: 2010, Vitamin A as a regulator of antigen presenting cells. J Nutr 140:1395-1399.

8. Eichner R: 1986, Epidermal effects of retinoids: in vitro studies. J Am Acad Dermatol 15:789-797.

9. Elias PM: 1986, Epidermal effects of retinoids: supramolecular observations and clinical implications. J Am Acad Dermatol 15:797-809.

10. Elias PM, Fritsch PO, Lampe M, et al.: 1981, Retinoid effects on epidermal structure, differentiation, and permeability. Lab Invest 44:531-540.

11. Fisher C, Blumenberg M, Tomić-Canić M: 1995, Retinoid receptors and keratinocytes. Crit Rev Oral Biol Med 6:284-301.
12. Fisher GJ, Voorhees JJ: 1996, Molecular mechanisms of retinoid actions in skin. FASEB J 10:1002-1013.

13. Fu PP, Xia Q, Boudreau MD, et al.: 2007, Physiological role of retinyl palmitate in the skin. Vitam Horm 75:223-256.

14. Ginn PE, Mansell JE, Rakich PM: 2007, Skin and appendages. In: Jubb, Kennedy, and Palmer's pathology of domestic animals, vol. 1, ed. Maxie MG, 5th ed., p. 628. Elsevier, Philadelphia, PA.

15. Hill B, Holroyd R, Sullivan M: 2009, Clinical and pathological findings associated with congenital hypovitaminosis A in extensively grazed beef cattle. Aust Vet J 87:94-98.

16. Jean J, Soucy J, Pouliot R: 2011, Effects of retinoic acid on keratinocyte proliferation and differentiation in a psoriatic skin model. Tissue Eng Part A 17:1859-1868.

17. Jones TC, Hunt RD, King NW: 1997, Diseases due to extraneous poisons. In: Veterinary pathology, 6th ed., pp. 748-751. Lippincott Williams \& Wilkins, Baltimore, MD.

18. Lanska DJ: 2010, Chapter 29: historical aspects of the major neurological vitamin deficiency disorders: overview and fatsoluble vitamin A. Handb Clin Neurol 95:435-444.

19. Panciera RJ, McKenzie DM, Ewing PJ, Edwards WC: 1993, Bovine hyperkeratosis: historical review and report of an outbreak. Compend Contin Educ Pract Vet 15:1287-1294.

20. Paulsen ME, Johnson L, Young S, et al.: 1989, Blindness and sexual dimorphism associated with vitamin A deficiency in feedlot cattle. J Am Vet Med Assoc 194:933-937.

21. Radostits OM, Gay CC, Hinchcliff KW, Constable PD: 2007, Diseases associated with nutritional deficiencies. In: Veterinary medicine, a textbook of the diseases of cattle, horses, sheep, pigs and goats, 10th ed., pp. 1771-1777. Elsevier, Philadelphia, PA.

22. Thompson K: 2007, Bones and joints. In: Jubb, Kennedy, and Palmer's pathology of domestic animals, vol. 1, ed. Maxie MG, 5th ed., pp. 55-56. Elsevier, Philadelphia, PA. 